\title{
Energies of the first row atoms from quantum Monte Carlo
}

\author{
M.D. Brown, J.R. Trail, P. López Ríos, and R.J. Needs \\ Theory of Condensed Matter Group, Cavendish Laboratory, \\ J.J. Thomson Avenue, Cambridge, CB3 OHE, UK
}

(Dated: June, 2007)

\begin{abstract}
All-electron variational and diffusion quantum Monte Carlo calculations of the ground state energies of the first row atoms ( $\mathrm{Li}$ to $\mathrm{Ne}$ ) are reported. We use trial wavefunctions of four types: single determinant Slater-Jastrow wavefunctions; multi-determinant Slater-Jastrow wavefunctions; single determinant Slater-Jastrow wavefunctions with backflow transformations; multi-determinant Slater-Jastrow wavefunctions with backflow transformations. At the diffusion quantum Monte Carlo level and using our best trial wavefunctions we recover $99 \%$ or more of the correlation energy for $\mathrm{Li}, \mathrm{Be}, \mathrm{B}, \mathrm{C}, \mathrm{N}$, and Ne, $97 \%$ for $\mathrm{O}$, and $98 \%$ for $\mathrm{F}$.

PACS numbers:
\end{abstract}

\section{INTRODUCTION}

Quantum Monte Carlo (QMC) methods can yield highly accurate energies for atoms, molecules and solids 1,2 , offering an alternative to quantum chemistry approaches such as Configuration Interaction and Coupled Cluster ${ }^{3}$. Although the cost of QMC calculations is large, the scaling with system size is much better than for accurate quantum chemistry approaches, and QMC methods have been applied to systems with of order $10^{3}$ electrons ${ }^{4}$.

The two most commonly used continuum QMC methods are variational quantum Monte Carlo (VMC) and diffusion quantum Monte Carlo (DMC). Stochastic integration is used in VMC to evaluate expectation values with a trial wavefunction, to a precision which can be systematically improved by increasing the number of configurations sampled. DMC is a stochastic projector method in which a wavefunction is evolved in imaginary time towards the ground state. Exact projector methods suffer from a fermion sign problem, and to overcome this we use the standard fixed-node approximation ${ }^{5,6}$ in which the nodal surface of the wavefunction is fixed during the evolution. The fixed-node approximation is uncontrolled, but the DMC energy is lower than the VMC energy with the same input trial wavefunction, and both give upper bounds on the exact energy.

DMC gives the lowest energy consistent with the fixed nodal surface. It is therefore advantageous to optimize parameters in the trial wavefunction, which may improve the trial nodal surface. The simplest and most widelyused trial wavefunction is the Slater-Jastrow (SJ) form, which consists of a Slater determinant of single-particle orbitals multiplied by a Jastrow correlation factor. More advanced wavefunctions can be obtained by, for example, replacing the single determinant by a sum over configuration state functions (CSFs), by using backflow transformations of the electronic coordinates,,$\underline{\underline{7}-10}$, or by using pairing wavefunctions ${ }^{11}-13$.

We present results of QMC calculations for the first row atoms ( $\mathrm{Li}$ to $\mathrm{Ne}$ ) obtained using trial wavefunctions of single and multi-determinant forms constructed from orbitals calculated on radial grids, both with and without backflow transformations. Our aims are to assess the different roles of multi-determinants and backflow in improving the trial wavefunctions for the first row atoms and to obtain benchmark VMC and DMC energies for them. We find that the use of multiple CSFs and backflow transformations recovers a substantial additional fraction of the correlation energy at both the VMC and DMC levels. Our best wavefunctions recover $99 \%$ or more of the correlation energy at the DMC level for $\mathrm{Li}, \mathrm{Be}, \mathrm{B}, \mathrm{C}, \mathrm{N}$, and $\mathrm{Ne}$, and more than $97 \%$ for $\mathrm{O}$ and $98 \%$ for $\mathrm{F}$. This represents a substantial advance upon previous DMC calculations.

\section{WAVEFUNCTION FORM AND OPTIMIZATION}

Our multi-CSF SJ wavefunctions with backflow transformations can be written in the form

$$
\Psi\left(\left\{\mathbf{r}_{i}\right\}, \mathbf{p}\right)=e^{J\left(\left\{\mathbf{r}_{i}\right\}, \mathbf{b}\right)} \sum_{n=1}^{N_{\mathrm{CSF}}} \alpha_{n} \Phi_{n}\left(\left\{\mathbf{x}_{i}\right\}\right),
$$

where each $\mathbf{x}_{i}=\mathbf{x}_{i}\left(\left\{\mathbf{r}_{i}\right\}, \mathbf{c}\right)$ results from the backflow transformation of the electronic coordinates $\left\{\mathbf{r}_{i}\right\}$, each $\Phi_{n}$ is a multideterminant representation of a CSF, and $\mathbf{p}=(\mathbf{a}, \mathbf{b}, \mathbf{c})$ are the variable wavefunction parameters: the CSF coefficients $\mathbf{a}$, the Jastrow parameters $\mathbf{b}$, and the backflow parameters $\mathbf{c}$. It is worth noting that the Jastrow factor normally introduces spin-contamination 14 and the backflow transformation angular momentum contamination into the trial wavefunction, so that $\Psi$ will not normally be a spin/spatial angular momentum eigenstate. However, the VMC and DMC energies still provide upper bounds on the true ground state energies.

The general form of our Jastrow factor $J\left(\left\{\mathbf{r}_{i}\right\}, \mathbf{b}\right)$ is described in an earlier paper $\frac{15}{5}$. In this study we used Jastrow factors containing electron-electron, electronnucleus, and electron-electron-nucleus terms. Our backflow transformations also consisted of electron-electron, electron-nucleus, and electron-electron-nucleus terms, as 
described in Ref $\stackrel{7}{7}$. For both the Jastrow and backflow functions, the expansion orders and spin dependencies were chosen by increasing the variational freedom until no further lowering of the VMC energy was observed. The maximum number of variable parameters in the trial wavefunctions varied from 146 for Li to 252 for B, N, C, $\mathrm{O}$, and $\mathrm{F}$. The number of variable parameters is therefore only weakly dependent on the atomic number.

We used the multiconfigurational Hartree-Fock (MCHF) atomic-structure package ATSP2K ${ }^{16}$ to construct the $\Phi_{n}$ functions appearing in Eq. (1). This provides the lowest energy solution of the many-electron problem where the solution is limited to an 'active space' (AS) specified by an allowed set of subshell occupation numbers, or configurations. The wavefunction may formally be written as a sum of CSFs,

$$
|L S\rangle_{\mathrm{AS}}=\sum_{\nu}^{\mathrm{AS}} a_{\nu}|\nu L S\rangle,
$$

where $\nu$ specifies a configuration (for example, $1 s^{2} 2 s^{2} 2 p^{3}$ ) and seniority number $\frac{17}{}$. Each CSF, $|\nu L S\rangle$, is defined by a radial orbital for each subshell, and by the total spatial/spin angular momentum quantum numbers, usually denoted ${ }^{2 S+1} L$.

An additional freedom exists because the underlying symmetry of the Hamiltonian results in degenerate solutions corresponding to different projection angular momentum eigenvalues, $L_{z}$ and $S_{z}$. These additional quantum numbers are not required to specify the total energy, the coefficients $a_{\nu}$, and the mean field equations for the radial orbitals, and they are not provided by the ATSP2K code. As a result it is not straightforward to obtain an explicit form for the CSFs which is suitable for use in QMC calculations. For the calculations presented here an appropriate representation of each $\mathrm{CSF}, \Phi_{n}$, was constructed as follows.

First we note that two separate angular momentum eigenstates containing $n_{1}$ and $n_{2}$ electrons may be coupled together to generate angular momentum eigenstates containing $n_{1}+n_{2}$ electrons $\frac{17}{}$. Starting with single electrons and performing such a coupling iteratively for every allowed combination provides all of the total spin/angular momentum eigenstates possible for a given configuration as sums of determinants of spin-orbitals. Due care was taken to avoid redundant computation, to respect antisymmetry, and to employ the same coupling order/sign conventions $\frac{18}{18}$ as used in ATSP2K, allowing the efficient construction of each CSF as a sum of determinants of one-electron spin-orbitals.

A different wavefunction results from each choice of $L_{z}$ and $S_{z}$. The spin independence of the Hamiltonian allows a direct conversion of the sum of determinants of complex spin-orbitals into a sum of products of two determinants of real orbitals, one for spin up and one for spin down electrons. This procedure provides a representation of

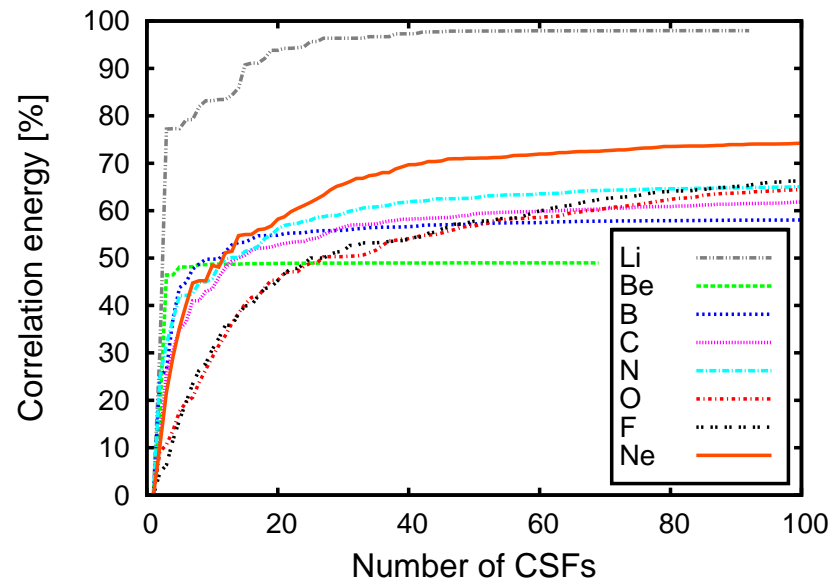

FIG. 1: (Color online) The percentage of the correlation energy recovered for each atom as a function of the number of CSFs included in the MCHF wavefunction. Core excitations were included for Li but not for the other atoms.

each CSF in the form

$$
\Phi_{\nu L S}=\sum_{j} a_{j} D_{j}^{\uparrow}(\nu L S) D_{j}^{\downarrow}(\nu L S),
$$

where the determinants are of real single-particle orbitals, and such that $\sum_{\nu} a_{v} \Phi_{\nu L S}$ has the same energy expectation value as the original MCHF wavefunction. Note that although $\Phi_{\nu L S}$ has total spatial angular momentum $L$ and projected spin angular momentum $S_{z}$, it is not necessarily an eigenstate of $L_{z}$ or $S^{2}$.

It should be clear that the $\Phi_{n}$ are not uniquely specified by $\left(\nu,{ }^{2 S+1} L\right)$ due to the spherical symmetry of the underlying Hamiltonian. In what follows we make the specific choice $\left|L_{z}\right|=L$, as this provides the smallest multideterminant expansions, and $\left|S_{z}\right|=S$, as this provides that same number of spin up/down electrons as predicted by Hund's first rule.

To define the multideterminant part of Eq. (1) we take ${ }^{2 S+1} L$ from the lowest energy HF state, and define an AS using the following rules for allowed excitations from the HF ground state configuration: only single and double (SD) electronic excitations were allowed, excitations of the $1 s$ core electrons were not allowed, and excitations were allowed into orbitals with quantum numbers $n \leq 7$ and $l \leq 5$. A MCHF calculation was then performed for the chosen AS, and the resulting CSFs ordered by weight, $a_{v}^{2}$. All but the $N_{C S F}$ largest-weight configurations were then discarded, and a MCHF calculation was performed using this reduced $\mathrm{AS}\left(N_{C S F}\right)$ active space. The percentages of the correlation energies recovered at the MCHF level are shown in Fig. 1 for $N_{C S F} \leq 100$.

$\mathrm{Li}$ is an exception: the above rules define an active space that is equivalent to HF because of the absence of $1 s$ excitations. In order to generate a nontrivial AS for $\mathrm{Li}$ we allowed excitations of all electrons into orbitals with quantum numbers $n \leq 5$ and $l \leq 5$. 


\begin{tabular}{|c|c|c|c|c|c|c|c|c|}
\hline & $\mathrm{Li}\left({ }^{2} \mathrm{~S}\right)$ & $\mathrm{Be}\left({ }^{1} \mathrm{~S}\right)$ & $\mathrm{B}\left({ }^{2} \mathrm{P}\right)$ & $\mathrm{C}\left({ }^{3} \mathrm{P}\right)$ & $\mathrm{N}\left({ }^{4} \mathrm{~S}\right)$ & $\mathrm{O}\left({ }^{3} \mathrm{P}\right)$ & $\mathrm{F}\left({ }^{2} \mathrm{P}\right)$ & $\mathrm{Ne}\left({ }^{1} \mathrm{~S}\right)$ \\
\hline & $.47683(3)$ & $1(1)$ & $24.6056(2)$ & $17(1)$ & 3) & -75 & 3) & -1 \\
\hline & $.47792(2)$ & $6(1)$ & 24.6 & 1) & 1) & 2) & (2) & - \\
\hline VM & $-7.47799(1)$ & -14.66 & -24.6 & $130(8)$ & $-54.5840(1)$ & -75 . & $-99.7220(2)$ & $9246(4)$ \\
\hline $\mathrm{DMC}$ & $7.47802(1)$ & $-14.65717(7)$ & -24.6 & $-37.8295(1)$ & -54.576 & -75 & $-99.7167(8)$ & $223(4)$ \\
\hline IDBF & $060(2)$ & -14.6 & -24.6 & -37 & -54 & -75 & $77(2)$ & $9339(3)$ \\
\hline DMC2 & $-7.478058(2)$ & -14.66 & -24.65 & -37.8 & -54.587 & -75 & $274(2)$ & $9339(3)$ \\
\hline$E_{\mathrm{HF}}$ & -7.432727 & -1 & -24.529061 & -37 & -54 & -74 & -99 & -12 \\
\hline & $-7.47806032^{a}$ & $-14.66736^{b}$ & $-24.65391^{b}$ & -37 & $-54.5892^{b}$ & -75 & $-99.7339^{b}$ & $9376^{b}$ \\
\hline$E_{\mathrm{HF}}-E_{\mathrm{ex}}$ & 0.04533332 & 0.094337 & 0.124849 & 0.156381 & 0.18826 & 0.257902 & 0.32455 & 0.390502 \\
\hline
\end{tabular}

TABLE I: The VMC and DMC energies (in Hartrees) for each atom, using a single determinant SJ wavefunction, a single determinant SJ wavefunction with backflow (BF), multiple determinants (MD), and both together (MDBF). The DMC2-MDBF results are for a second DMC run with half the timestep of DMC-MDBF. Also shown are the Hartree-Fock single determinant energies $\left(E_{\mathrm{HF}}\right)$, the "exact" energies $E_{\text {exact }}$, the correlation energies $E_{\mathrm{HF}}-E_{\text {exact }}$, and the percentage of the correlation energy recovered by the DMC2-MDBF calculations (DMC2-corr\%). The numbers in parentheses indicate the statistical uncertainty in the last digit shown.

${ }^{a}$ taken from Ref: ${ }^{22}$ (rounded to 9 significant figures).

${ }^{b}$ taken from Ref. ${ }^{23}$.

We chose $N_{C S F}=20$ for the multideterminant expansions used in the QMC calculations (corresponding to a number of determinants ranging from 83 for Li to 499 for $\mathrm{Ne})$. Although the application of this selection criterion to the first row atoms is necessarily somewhat arbitrary, it is not without justification. Figure 1 shows that for around 20 CSFs the fraction of correlation energy recovered within $\mathrm{MCHF}$ is roughly equivalent from Be to Ne. Including further orbitals in the initial 'large' AS, including triple excitations, or allowing core excitations, did not significantly change the MCHF energies for $N_{C S F} \leq 20$, with the exceptions of $\mathrm{Li}$ and $\mathrm{Be}$ for which core excitations improved the MCHF energies significantly for small $N_{C S F}$, but had a negligible impact on the DMC results.

The variable parameters $\mathbf{p}$ were optimized by minimizing the VMC energy, using the scheme developed very recently by Umrigar et al $\underline{19,20}$. This scheme is a generalisation of the method of Nightingale and MelikAlaverdian $\underline{21}$ to include non-linear parameters in the trial wavefunction. In the past, variance minimization techniques were normally used because they are robust for optimizing Jastrow factors, but in fact they have been found to behave poorly when optimizing linear coefficients. The method of Nightingale and Melik-Alaverdian ${ }^{21}$ is very robust for optimizing linear coefficients, and Umrigar et $a l, \underline{19}, 20$ have developed an energy minimization scheme which is robust for both linear and non-linear parameters. All of the wavefunction parameters were optimized simultaneously in our calculations. In some cases we obtained significantly lower VMC and DMC energies with energy minimization than with variance minimization when optimizing the CSF coefficients $\mathbf{a}$, and the performance for the Jastrow and backflow parameters (b and $\mathbf{c}$ ) was also slightly improved.

\section{RESULTS}

We used the CASINO code ${ }^{24}$ for all of our VMC and DMC calculations. The wavefunction optimizations were performed using $10^{5}$ statistically independent electronic configurations. The target population of walkers in the DMC calculations was about 2000 in each case, which

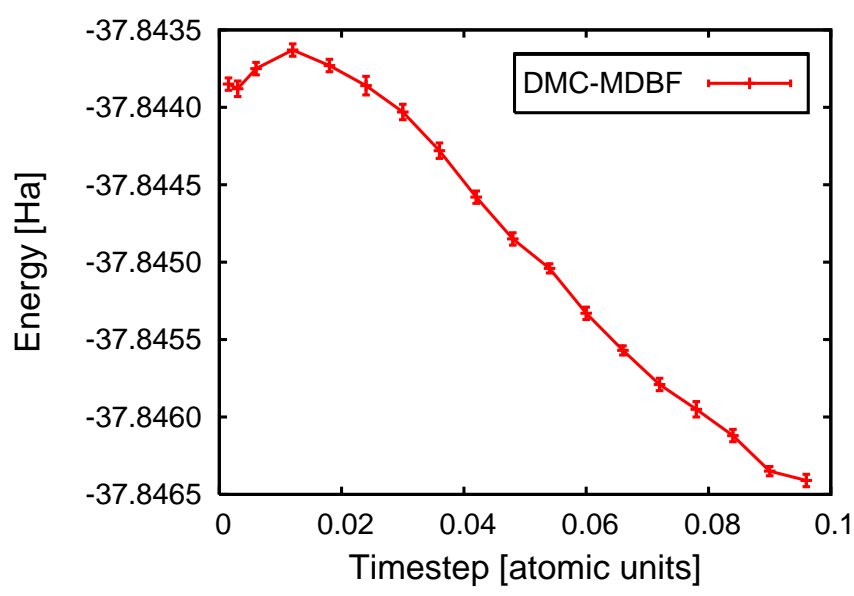

FIG. 2: (Color online) The variation of the DMC energy (in Hartrees) with timestep for the $\mathrm{C}$ atom, using a MDBF wavefunction. The statistical error bars on the data are shown. 


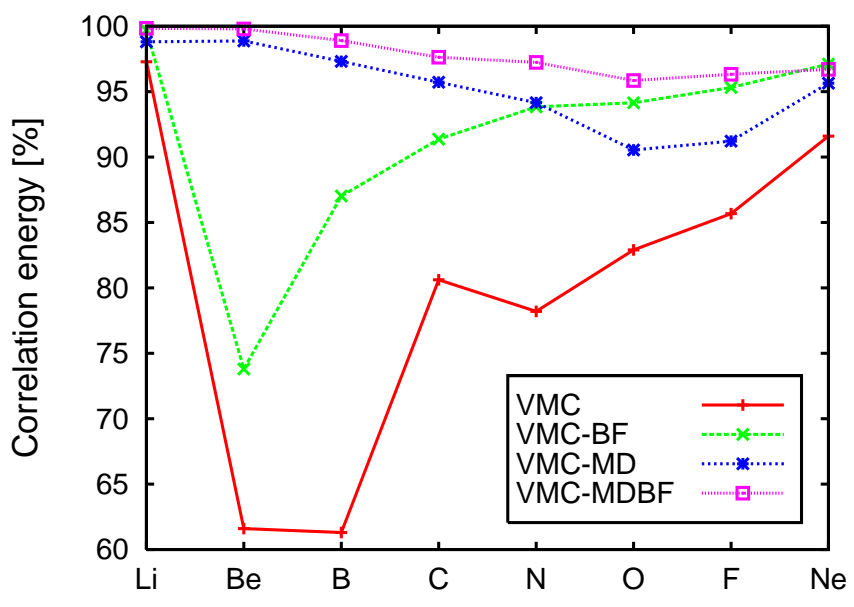

FIG. 3: (Color online) The percentage of the correlation energy recovered for each atom within VMC, using a single determinant SJ wavefunction (VMC) with the addition of backflow (VMC-BF), multiple determinants (VMC-MD), and both together (VMC-MDBF). The statistical error bars (not shown) are smaller than the symbols.

should ensure that any population control bias is negligible. The timesteps for the DMC calculations were chosen to make the systematic finite timestep errors smaller than the random statistical errors. The smallest timesteps used, corresponding to the DMC2 data in Table】 ranged from 0.00375 a.u. for Li to 0.0007 a.u. for Ne. The variation of the DMC energy of the $\mathrm{C}$ atom with timestep is shown in Fig. 2, and the shape of this curve is typical of our calculations for the first row atoms. For C, the DMC acceptance ratios varied from $93(1) \%$ for the largets time step (0.096 a.u.) to $99.8(1) \%$ for the smallest time step (0.0015 a.u.).

Table I gives the VMC and DMC energies, including the most accurate correlation energy percentages achieved for each atom. The "exact" non-relativistic energies, assuming a point nucleus of infinite mass, were taken from Refs $\frac{22,23}{2}$. Fig. 3 shows the percentage of each atom's correlation energy recovered by the optimized wavefunctions within VMC as the multi-CSF expansion and backflow transformations were added. Fig. [4 gives the same information at the DMC level.

The general shapes of the curves in Figs. 3 and 4 are similar, but note that the VMC graph covers a range of $40 \%$, while the DMC one covers only $12 \%$. Our best VMC results surpass the accuracy previously achieved in DMC calculations using single determinant SJ trial wavefunctions ${ }^{25-27}$. Our single determinant DMC results are similar to those reported by other authors $25-27$. For $\mathrm{Li}$ and $\mathrm{Be}$ our best DMC energies are within error bars of some previous DMC calculations, but for the other atoms our results are clearly superior. Flad et al. $\frac{28}{2}$ performed multideterminant DMC calculations for the atoms B to $\mathrm{F}$, but we have used larger expansions and our results are already superior at the DMC-MD level. Casula and
Sorella 11 performed DMC calculations for the atoms Li$\mathrm{Ne}$ with correlated geminal pairing wavefunctions, but our energies are substantially better than theirs. VMC and DMC calculations using backflow wavefunctions have been reported for $\mathrm{Li}^{7}$ and $\mathrm{Ne}^{8}$. Lüchow and Fink ${ }^{29}$ performed a DMC calculation for $\mathrm{N}$ using a pair natural orbital configuration interaction trial wavefunction, retrieving $98.2 \%$ of the correlation energy, which is very similar to our DMC-MD result, but our DMC-MDBF energy is lower. Our results for $\mathrm{Li}$ and Be are surpassed by Hylleraas variational calculations ${ }^{22,30}$. We should also note the existence of high quality non-variational results such as those from explicitly correlated $r_{12}$ Configuration Interaction and Coupled Cluster methods 31,32 .

Each of the VMC and DMC energies for Li corresponds to recovering well over $90 \%$ of the correlation energy. The single-determinant HF nodal surface of $\mathrm{Li}$ is extremely accurate, but incorporating backflow improves the DMC energy noticeably. Backflow gives a significant improvement for Be at the VMC level, but a very modest improvement within DMC. The ground state wavefunction for Be contains a substantial admixture of the $1 s^{2} 2 p^{2}$ excited state configuration which significantly alters the nodal surface. The single determinant $1 s^{2} 2 s^{2}$ HF nodal surface divides the configuration space into four nodal pockets, while the exact wavefunction has only two nodal pockets 33,34 . It does not appear possible to achieve an accurate DMC energy for Be with a trial wavefunction containing four nodal pockets. The continuous backflow transformations we use are incapable of changing the number of nodal pockets ${ }^{\underline{7}}$ and consequently the single determinant DMC energy for Be is very poor. When an appropriate amount of the $1 s^{2} 2 p^{2}$ configuration is included in the trial wavefunction, the DMC energy improves dra-

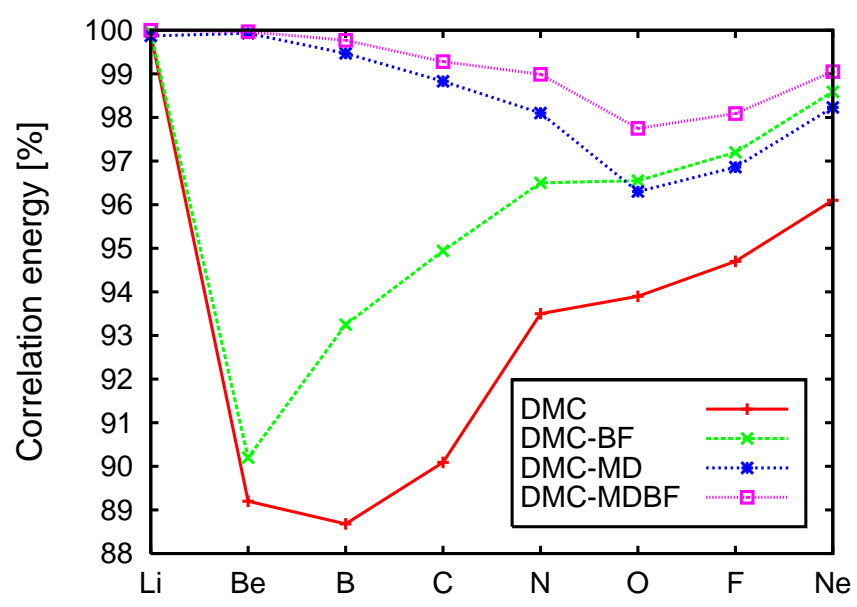

FIG. 4: (Color online) The percentage of the correlation energy recovered for each atom within DMC, using a single determinant SJ wavefunction (DMC) with the addition of backflow (DMC-BF), multiple determinants (DMC-MD), and both together (DMC-MDBF). The statistical error bars (not shown) are smaller than the symbols. 
matically, and backflow contributes a small additional improvement.

The percentages of the correlation energy recovered for $\mathrm{N}$ at the VMC level from the single determinant backflow and $20 \mathrm{CSF}$ wavefunctions are almost the same, while for higher atomic numbers backflow gives more correlation energy than the CSFs. Fig. 1 1 shows that the percentages of the correlation energy recovered within the MCHF calculations for $\mathrm{O}$ and $\mathrm{N}$ increase relatively slowly with the number of CSFs, which may be connected with the lower percentages of correlation energy recovered in the $\mathrm{MD}$ and MDBF calculations for these atoms. Note that, apart from $\mathrm{Li}$ and Be where the DMC-MD energies with 20 CSFs are already excellent, backflow adds substantially to the correlation energy recovered. It seems that backflow and multi-CSFs improve the single determinant wavefunctions in rather different ways, and therefore it can be very advantageous to combine them.

One might expect that the VMC-MDBF energies would always be lower than the VMC-BF and VMC-MD energies, as the wavefunction parameters are obtained by minimizing the VMC energy. Table 1 shows, however, that this does not hold for Ne. The most likely reason for this is that the HF and MCHF orbitals are different, so that the single determinant wavefunction does not correspond to a particular choice of the MD wavefunction parameter values. This also indicates that further reductions in the VMC energy could be obtained by optimizing the orbitals along with the Jastrow and backflow parameters and the CSF coefficients.

\section{DISCUSSION}

As can be seen from Fig. 4, we recover $99 \%$ or more of the correlation energy for all the atoms except $\mathrm{O}$ and F. The different freedoms introduced by the multi-CSF expansion and backflow transformations are both vital in achieving the best energies, indicating that they capture different aspects of the wavefunction correlation. Backflow appears to describe parts of the dynamic correlation beyond the SJ wavefunction while the low-energy CSFs beyond the HF ground state configuration describe static correlation energy. Our results contribute to the growing evidence ${ }^{7,8}$ that adding backflow transformations to SJ wavefunctions can significantly improve the nodal surface for systems from atoms to solids.

It should be noted that, while it might be difficult to substantially improve the Jastrow factors and backflow transformations, we could easily include many more CSFs in the trial wavefunctions without a prohibitive increase in computational expense. The orbitals in the CSFs could also be optimized within VMC. The energies reported here do not therefore represent the current practical limits of the VMC and DMC methods for allelectron atoms.

For general systems, the number of CSFs required to obtain a significant improvement in the energy increases rapidly with the number of electrons $N$. The number of required parameters in the backflow functions only increases (roughly linearly) with the number of inequivalent aoms. The cost of moving an electron in a VMC/DMC calculation scales approximately as $N^{2}$. In a VMC-BF/DMC-BF calculation the cost scales as $N^{3}$ because all of the orbitals must be evaluated at the positions $\left\{\mathbf{x}_{i}\right\}$ when an electron is moved. The cost of a VMC-MD/DMC-MD calculation scales as $N^{2} M$, where $M$ is the number of determinants, and the cost of a VMC-MDBF/DMC-MDBF calculation scales as $N^{3} M$. The number of moves required to obtain a fixed statistical error bar in the energy is smaller for a more accurate trial wavefunction, so that the pre-factors in the cost for the more sophisticated wavefunction forms are smaller. As an example, for the $\mathrm{C}$ atom the relative computational costs to achieve the same error bar are 1.0/3.6/6.2/17.8 for the $\mathrm{VMC} \mathrm{SD} / \mathrm{SDBF} / \mathrm{MD} / \mathrm{MDBF}$ calculations, while in DMC the corresponding cost ratios are 9.0/13.7/15.1/31.9. Overall, we expect that we can maintain the level of accuracy achieved here for small molecules.

We obtained superior results with the energy minimization scheme of Umrigar et al $\underline{\underline{19}}$ than with variance minimization procedures ${ }^{35-37}$. The energy minimization method is particularly advantageous when multiple determinants are used because it is well-suited to optimizing linear parameters, whereas variance minimization encounters some difficulties in optimizing CSF coefficients. The combination of energy minimization and the MD and $\mathrm{BF}$ wavefunction forms has led to substantial reductions in the VMC energies, which are very important because the variance of the DMC energy has been found to be proportional to the error in the VMC energy ${ }^{38.39}$. Although reductions in the DMC energies from the improved trial wavefunctions are smaller than in VMC, they represent substantial fractions of the remaining correlation energy. Our best DMC energies for $\mathrm{Li}$ and Be are within error bars of previously published DMC energies, but our results for the higher atomic number atoms are markedly superior.

\section{Acknowledgments}

Financial support was provided by the Engineering and Physical Sciences Research Council (EPSRC) of the UK. Computing resources were provided by the University of Cambridge High Performance Computing Service (HPCS).
1 W.M.C. Foulkes, L. Mitas, R.J. Needs, and G. Rajagopal, Rev. Mod. Phys. 73, 33 (2001).
${ }^{2}$ C.J. Umrigar and M.P. Nightingale, eds., Quantum Monte 
Carlo Methods in Physics and Chemistry, NATO ASI Ser. C 525 (Kluwer, Dordrecht, 1999).

3 A. Szabo and N.S. Ostlund, Modern Quantum Chemistry (Dover, 1996).

4 R. Maezono, A. Ma, M.D. Towler and R.J. Needs, Phys. Rev. Lett. 98, 025701 (2007).

5 J.B. Anderson, J. Chem. Phys. 63, 1499 (1975).

${ }^{6}$ D.M. Ceperley and B.J. Alder, Phys. Rev. Lett. 45, 566 (1980).

7 P. López Ríos, A. Ma, N.D. Drummond, M.D. Towler and R.J. Needs, Phys. Rev. E 74, 066701 (2006).

8 N.D. Drummond, P. López Ríos, A. Ma, J.R. Trail, G.G. Spink, M.D. Towler and R.J. Needs, J. Chem. Phys. 124, 224104 (2006).

9 R.P. Feynman, Phys. Rev. 94, 262 (1954).

10 R.P. Feynman and M. Cohen, Phys. Rev. 102, 1189 (1956).

11 M. Casula and S. Sorella, J. Chem. Phys. 119, 6500 (2003).

12 M. Casula, C. Attaccalite and S. Sorella, J. Chem. Phys. 121, 7110 (2004).

${ }^{13}$ M. Bajdich, L. Mitas, G. Drobný, L.K. Wagner and K.E. Schmidt, Phys. Rev. Lett. 96, 130201 (2006).

14 C. Huang, C. Filippi and C.J. Umrigar, J. Chem. Phys. 108, 8838 (1998).

15 N.D. Drummond, M.D. Towler and R.J. Needs, Phys. Rev. B 70, 235119 (2004).

16 C. Froese Fischer, G. Tachiev, G. Gaigalas and M. Godefroid Comput. Phys. Commun. 176, 559 (2007). (http://atoms.vuse.vanderbilt.edu).

17 C. Froese Fischer, T. Brage and P. Jönsson, Computational Atomic Structure: An MCHF approach (IOP Publishing Ltd. 1997).

18 G. Gaigalas, Z. Rudzikas and C. Froese Fischer, At. Data Nucl. Tables 70, 1 (1998).

19 C.J. Umrigar, J. Toulouse, C. Filippi, S. Sorella and R.G. Hennig, Phys. Rev. Lett. 98, 110201 (2007).

20 J. Toulouse and C.J. Umrigar, J. Chem. Phys. 126, 084102 (2007).

21 M.P. Nightingale and V. Melik-Alaverdian, Phys. Rev. Lett. 87, 043401 (2001).
22 M. Puchalski and K. Pachucki, Phys. Rev. A 73, 022503 (2006).

23 S.J. Chakravorty, S.R. Gwaltney, E.R. Davidson, F.A. Parpia and C.F. Fischer, Phys. Rev. A 47, 3649 (1993).

24 R.J. Needs, M.D. Towler, N.D. Drummond and P. López Ríos, CASINO user's guide, version 2.0.0 (2006).

25 A. Lüchow and J.B. Anderson, J. Chem. Phys. 105, 7573 (1996).

26 P. Langfelder, S.M. Rothstein and J. Vrbik, J. Chem. Phys. 107, 8525 (1997).

27 K. Hongo, Y. Kawazoe and H. Yasuhara, Materials Transactions 47, 2612 (2006).

28 H. Flad, M. Caffarel and A. Savin, in Recent Advances in Quantum Monte Carlo Methods, edited by W.A. Lester, Jr. (World Scientific, Singapore, 1997).

29 A. Lüchow and R.F. Fink, J. Chem. Phys. 113, 8457 (2000).

30 J. Komasa, J. Rychlewski and K. Jankowski, Phys. Rev. A 65, 042507 (2002).

31 R.J. Gdanitz, J. Chem. Phys. 109, 9795 (1998).

32 J. Noga, P. Valiron and W. Klopper, J. Chem. Phys. 115, 2022 (2001).

33 W.A. Glauser, W.R. Brown, W.A. Lester, Jr., D. Bressanini, B.L. Hammond and M.L. Koszykowski, J. Chem. Phys. 97, 9200 (1992).

34 D. Bressanini, D.M. Ceperley and P.J. Reynolds, in Recent Advances in Quantum Monte Carlo Methods, 2nd ed. edited by W.A. Lester, Jr., S.M. Rothstein and S. Tanaka (World Scientific, Singapore, 2002).

35 C.J. Umrigar, K.G. Wilson and J.W. Wilkins, Phys. Rev. Lett. 60, 1719 (1988).

36 P.R.C. Kent, R.J. Needs and G. Rajagopal, Phys. Rev. B 59, 12344 (1999).

37 N.D. Drummond and R.J. Needs, Phys. Rev. B 72, 085124 (2005).

38 D.M. Ceperley, J. Stat. Phys. 43, 815 (1986).

39 A. Ma, N.D. Drummond, M.D. Towler and R.J. Needs, Phys. Rev. E 71, 066704 (2005). 